Bull. Soc. math. France

133 (1), 2005, p. 145-157

\title{
PERTE DE RÉGULARITÉ POUR LES ÉQUATIONS D'ONDES SUR-CRITIQUES
}

\author{
PAR Gilles Lebeau
}

\begin{abstract}
RÉsumé. - On prouve que le problème de Cauchy local pour l'équation d'onde sur-critique dans $\mathbb{R}^{d}, \square u+u^{p}=0, p$ impair, avec $d \geq 3$ et $p>(d+2) /(d-2)$, est mal posé dans $H^{\sigma}$ pour tout $\left.\sigma \in\right] 1, \sigma_{\text {crit }}\left[\right.$, où $\sigma_{\text {crit }}=d / 2-2 /(p-1)$ est l'exposant critique.

ABSTRACT (Loss of regularity for super critical wave equations). - We prove that the local Cauchy problem for the supercritical wave equation in $\mathbb{R}^{d}, \square u+u^{p}=0$, with $d \geq 3, p>3$ and $p>(d+2) /(d-2)$, is ill-posed in $H^{\sigma}$ for every $\left.\sigma \in\right] 1, \sigma_{c}[$, where $\sigma_{c}=d / 2-2 /(p-1)$ is the critical exponent.
\end{abstract}

\section{Introduction et résultats}

On s'intéresse au problème de Cauchy local pour l'équation des ondes non linéaire dans $\mathbb{R}^{d}$, avec $d \geq 3$ et $p$ entier impair,

$$
\begin{gathered}
\left(\partial_{t}^{2}-\triangle_{x}\right) u+u^{p}=0 \\
\left.u(0, x)=u_{0}(x), \quad \partial_{t} u(0, x)\right)=u_{1}(x) .
\end{gathered}
$$

Texte reçu le 4 avril 2003, révisé le 16 juin 2003, accepté le 28 août 2003.

Gilles Lebeau, Université de Nice Sophia-Antipolis, Laboratoire J.-A. Dieudonné, UMR $6621 \mathrm{du}$ CNRS, Parc Valrose 06108 Nice Cedex 2 (France)

E-mail : lebeau@math.unice.fr

Classification mathématique par sujets (2000). - 35L05, 35L15.

Mots clefs. - Analyse microlocale, équations d'ondes non-linéaires.

BULLETIN DE LA SOCIÉTÉ MATHÉMATIQUE DE FRANCE

(C) Société Mathématique de France

$0037-9484 / 2005 / 145 / \$ 5.00$ 
Les solutions de l'équation (1.1) vérifient formellement la conservation de l'énergie

$$
E(u)=\int \frac{1}{2}\left(\left|\partial_{t} u\right|^{2}+\left|\nabla_{x} u\right|^{2}\right)+\frac{u^{p+1}}{p+1} \mathrm{~d} x .
$$

Le problème de Cauchy le plus naturel pour (1.1) est donc de supposer les données à l'instant $t=0$ dans l'espace d'énergie

$$
u(0, x) \in \dot{H}^{1} \cap L^{p+1}, \quad \partial_{t} u(0, x) \in L^{2} .
$$

Rappelons que le problème de Cauchy (1.1) avec données dans l'espace d'énergie possède une longue histoire.

Lorsque l'exposant $p$ est sous-critique, c'est-à-dire vérifie $p+1<d^{*}$, où $d^{*}=2 d /(d-2)$ est l'exposant de l'injection de Sobolev $\dot{H}^{1} \subset L^{d^{*}}$ dans $\mathbb{R}^{d}$, les premiers résultats furent obtenus en dimension 3 par Jörgens [4] en 1961, le cas de la dimension quelconque ayant été résolu par Ginibre et Velo [2] en 1985, où ils prouvent que les inégalités de Strichartz impliquent que le problème de Cauchy pour (1.1) avec données dans l'espace d'énergie est bien posé globalement en temps.

Dans le cas critique $p+1=d^{*}$, le problème de l'existence globale a été résolu par Shatah et Struwe [8] (avec l'unicité dans un espace précisé adapté aux estimations de Strichartz).

Nous supposerons donc que l'exposant $p$ est au contraire sur-critique, c'està-dire vérifie

$$
p+1>d^{*}
$$

Dans ce cas, l'existence de solutions faibles globales en temps a été obtenu par Segal [7], Lions [6], et Strauss [9].

L'éventuelle unicité de ces solutions faibles et leur continuité par rapport aux données de Cauchy demeurent des problèmes ouverts.

Nous avons toutefois prouvé dans [5] qu'il est impossible de construire les solutions faibles de sorte qu'elles dépendent continuement de leurs données de Cauchy, uniformément sur la boule unité de l'espace d'energie (ce qui est le cas lorsque $p$ est sous-critique).

Remarque 1.1. - Dans toute la suite, on appelera solution faible de l'équation (1.1) une solution au sens des distributions construites par approximation de l'équation, méthode que nous rappelons en appendice. En particulier, il résulte des constructions rappelées au $\S 6$, que si les données de Cauchy coïncident dans un ouvert $\Omega$ avec des fonctions ne dépendant au plus que de deux variables, par vitesse finie de propagation, les solutions faibles coïncident dans le domaine d'influence de $\Omega$ avec l'unique solution du problème $2 \mathrm{D}$ associé, donc indépendamment du choix de la fonction de régularisation $f_{\varepsilon}$, et de la suite extraite choisie dans le procédé de construction des solutions faibles. 
Les inégalités de Strichartz entraînent toutefois que le problème de Cauchy local (1.1), avec données dans l'espace

$$
\left.u(0, x) \in H^{\sigma}, \partial_{t} u(0, x)\right) \in H^{\sigma-1}
$$

est localement bien posé pour $\sigma$ vérifiant

$$
\sigma>\sigma_{\text {crit }}, \quad \sigma_{\text {crit }}=\frac{d}{2}-\frac{2}{p-1} .
$$

L'objet de cet article est de prouver que le problème de Cauchy local (1.1) est mal posé dans $H^{\sigma}$ pour tout $\sigma$ vérifiant $\left.\sigma \in\right] 1, \sigma_{\text {crit }}[$; pour énoncer le résultat, nous introduisons les notations suivantes.

Soit $\sigma_{\text {sob }}$ l'exposant de l'injection de Sobolev $H^{\sigma_{\text {sob }}} \subset L^{p+1}$ :

$$
\sigma_{\mathrm{sob}}=\frac{d}{2}-\frac{d}{p+1}
$$

Comme $p$ est sur-critique, on a $1<\sigma_{\text {sob }}<\sigma_{\text {crit }}$. Soit $I(\sigma)$ la fonction définie sur $\left[1, \sigma_{\text {crit }}\right]$ par

$$
I(\sigma)=1 \operatorname{sur}\left[1, \sigma_{\mathrm{sob}}\right], \quad I(\sigma)=\frac{2 \sigma}{(p-1)\left(\frac{1}{2} d-\sigma\right)} \operatorname{sur}\left[\sigma_{\mathrm{sob}}, \sigma_{\mathrm{crit}}\right] .
$$

On a $I(\sigma)<\sigma$ pour tout $\sigma \in] 1, \sigma_{\text {crit }}[$.

Le résultat qui suit exprime la perte de régularité des solutions faibles par rapport à la régularité de leurs données de Cauchy.

ThÉorème 1. - Pour tout $\sigma \in] 1, \sigma_{\text {crit }}\left[\right.$ et tout $\sigma^{\prime}>I(\sigma)$, il existe un couple de données de Cauchy $u_{0} \in H_{0}^{\sigma} \cap L^{p+1}, u_{1} \in C_{0}^{\infty}$, et une suite $t_{k}$ de limite nulle, telles que pour toute solution faible $u(t, x)$ de $(1.1),(1.2)$, on ait

$$
\lim _{k \rightarrow \infty}\left\|u\left(t_{k}, .\right)\right\|_{H^{\sigma^{\prime}}}=+\infty
$$

Remarque 1.2. - Il résultera de la construction qu'on peut choisir $u_{0} \in C_{0}^{\infty}$ en dehors de l'origine.

Soit $J(\sigma)$ la fonction définie sur $\left[1, \sigma_{\text {crit }}\right]$ par

$$
J(\sigma)=\frac{\frac{1}{2} d+2(\sigma-1) /(p-1)}{\frac{1}{2} d-(\sigma-1)} .
$$

ThÉORÈme 2. - Pour tout $\sigma \in] 1, \sigma_{\text {crit }}\left[\right.$ et tout $\sigma^{\prime}>J(\sigma)$, il existe un couple de données de Cauchy $u_{0} \in C_{0}^{\infty}, u_{1} \in H_{0}^{\sigma-1}$ et une suite $t_{k}$ de limite nulle, telles que pour toute solution faible $u(t, x)$ de (1.1), (1.2), on ait

$$
\lim _{k \rightarrow \infty}\left\|\partial_{t} u\left(t_{k}, .\right)\right\|_{H^{\sigma^{\prime}-1}}=+\infty
$$

BULlETIN DE LA SOCIÉTÉ MATHÉMATIQUE DE FRANCE 
Remarque 1.3. - On peut interpréter le théorème 2 comme une explosion instantannée des solutions faibles dans tout espace d'interpolation entre l'espace d'énergie et l'espace optimal des inégalités de Strichartz associé à l'homogénéité de l'équation.

\section{Homogénéité}

Soient $d^{\prime}>0$ et $d^{\prime \prime}>0$ tels que $d^{\prime}+d^{\prime \prime}=d$. Pour $x \in \mathbb{R}^{d}$, on pose $x=\left(x^{\prime}, x^{\prime \prime}\right)$ avec $x^{\prime} \in \mathbb{R}^{d^{\prime}}$ et $x^{\prime \prime} \in \mathbb{R}^{d^{\prime \prime}}$. Soient $\alpha>0$ et $\left.\left.\gamma \in\right] 0,1\right]$. Pour $\varphi \in C_{0}^{\infty}\left(\mathbb{R}^{d}\right)$ et $h \in] 0,1]$, on pose

$$
\varphi_{h}\left(x^{\prime}, x^{\prime \prime}\right)=h^{-\alpha} \varphi\left(\frac{x^{\prime}}{h}, \frac{x^{\prime \prime}}{h^{\gamma}}\right) .
$$

En désignant par $\widehat{\varphi}$ la transformation de Fourier et

$$
\delta=\frac{1}{2}\left(d^{\prime}+d^{\prime \prime} \gamma\right)
$$

on a

$$
\widehat{\varphi}_{h}\left(\xi^{\prime}, \xi^{\prime \prime}\right)=h^{2 \delta-\alpha} \widehat{\varphi}\left(h \xi^{\prime}, h^{\gamma} \xi^{\prime \prime}\right)
$$

d'où pour tout $\sigma$

$$
\begin{aligned}
\int\left(1+|\xi|^{2}\right)^{\sigma}\left|\widehat{\varphi}_{h}(\xi)\right|^{2} \mathrm{~d} \xi & \\
= & h^{2(\delta-\alpha-\sigma)} \int\left(h^{2}+\left|\xi^{\prime}\right|^{2}+h^{2(1-\gamma)}\left|\xi^{\prime \prime}\right|^{2}\right)^{\sigma}|\widehat{\varphi}(\xi)|^{2} \mathrm{~d} \xi
\end{aligned}
$$

Il en résulte

$$
\left\|\varphi_{h} ; H^{\sigma}\right\|=h^{\delta-\alpha-\sigma}\left(\int\left(h^{2}+\left|\xi^{\prime}\right|^{2}+h^{2(1-\gamma)}\left|\xi^{\prime \prime}\right|^{2}\right)^{\sigma}|\widehat{\varphi}(\xi)|^{2} \mathrm{~d} \xi\right)^{1 / 2} .
$$

La norme $L^{p+1}$ de $\varphi_{h}$ est quant à elle donnée par

$$
\left\|\varphi_{h} ; L^{p+1}\right\|=h^{2 \delta /(p+1)-\alpha}\left\|\varphi ; L^{p+1}\right\| .
$$

Si on effectue le changement d'échelle

$$
x^{\prime}=h y^{\prime}, \quad x^{\prime \prime}=h^{\gamma} y^{\prime \prime}, \quad u\left(t, x^{\prime}, x^{\prime \prime}\right)=h^{-\alpha} v\left(t, y^{\prime}, y^{\prime \prime}\right),
$$

$u$ sera solution de (1.1) si, et seulement si, $v$ est solution de l'équation

$$
h^{(p-1) \alpha} \partial_{t}^{2} v-h^{2 \beta}\left(\triangle_{y^{\prime}}+h^{2(1-\gamma)} \triangle_{y^{\prime \prime}}\right) v+v^{p}=0
$$

avec

$$
\beta=\frac{(p-1) \alpha}{2}-1 \text {. }
$$

La stratégie de construction de solutions singulières $u$ pour (1.1) va consister à construire d'abord des solutions $v$ de (2.8) et à définir ensuite $u$ comme une superposition de solutions définies par (2.7). Comme on veut contrôler les normes $H^{\sigma}$ et $L^{p+1}$ de la donnée $u(0, x)$, et qu'il semble judicieux de faire en 
sorte que (2.8) ne dégénère pas sur une équation des ondes linéaires lorsque $h$ tend vers 0 , on impose les relations déduites de (2.5), (2.6) et (2.8)

$$
\alpha=\min \left(\delta-\sigma, \frac{2 \delta}{p+1}\right), \quad \beta>0 .
$$

Lemme 2.1. - Pour tout $\sigma \in\left[1, \sigma_{\text {crit }}\left[\right.\right.$, les solutions $\delta \leq \frac{1}{2} d$ aux inéquations (2.10) sont les réels

$$
\left.\delta \in] \sigma+\frac{2}{p-1}, \frac{d}{2}\right] .
$$

Démonstration. — Les inéquations (2.10) signifient

$$
\min \left(\delta-\sigma, \frac{2 \delta}{p+1}\right)>\frac{2}{p-1}
$$

c'est-à-dire $\delta>\sigma+2 /(p-1)$ pour $\delta \leq \sigma(p+1) /(p-1)$ et $\delta>(p+1) /(p-1)$ pour $\delta \geq \sigma(p+1) /(p-1)$, d'où le résultat.

Remarque 2.1. - La quantité $2 \delta$ joue le rôle d'une dimension virtuelle pour le changement d'échelle (2.7), de sorte que la borne inférieure dans (2.11) est exactement la valeur critique (1.7) associée à la dimension $2 \delta$.

\section{3. Équation en dimension 1}

On s'intéresse dans cette partie à l'équation en dimension 1 , avec $s, z \in \mathbb{R}$,

$$
\partial_{s}^{2} v-\partial_{z}^{2} v+v^{p}=0, \quad v(0, z)=v_{0}, \quad \partial_{s} v(0, z)=v_{1}
$$

On notera $G(s)$ la solution de l'équation différentielle ordinaire

$$
G^{\prime \prime}+G^{p}=0, \quad G(0)=1, G^{\prime}(0)=0 .
$$

Soit $L$ le linéarisé de (3.1) sur la solution $G(s)$ indépendante de $z$,

$$
L(w)=\partial_{s}^{2} w-\partial_{z}^{2} w+p G^{p-1} w .
$$

Soient $\Gamma$ la période de la fonction $G$ et $M(\lambda)$ la matrice de transfert associée à l'équation de Hill sur $\mathbb{R}, f^{\prime \prime}+p G^{p-1} f$

$$
\left(\begin{array}{c}
f(\Gamma) \\
f^{\prime}(\Gamma)
\end{array}\right)=M(\lambda)\left(\begin{array}{c}
f(0) \\
f^{\prime}(0)
\end{array}\right), \quad f^{\prime \prime}+p G^{p-1} f+\lambda f=0 .
$$

On a det $M(\lambda)=1$ et les valeurs propres de $M(\lambda)$ sont réelles si, et seulement si, $|\operatorname{tr}(M(\lambda))| \geq 2$.

Soit $Z(s)$ l'opérateur reliant les données de Cauchy des solutions de (3.3) entre les instants 0 et $s$. Par définition de $M$, on a

$$
Z(\Gamma)\left(\begin{array}{l}
w_{0} \\
w_{1}
\end{array}\right)=\frac{1}{2 \pi} \int \mathrm{e}^{i z \zeta} M\left(\zeta^{2}\right)\left(\begin{array}{l}
\widehat{w}_{0}(\zeta) \\
\widehat{w}_{1}(\zeta)
\end{array}\right) \mathrm{d} \zeta
$$

où $\widehat{f}(\zeta)=\int \mathrm{e}^{-i z \zeta} f(z) \mathrm{d} z$ est la transformée de Fourier en $z$. 
Proposition 3.1. - (i) On a $\operatorname{tr}(M(\lambda)) \geq-2$ pour tout $\lambda$.

(ii) L'ensemble d'instabilité $I=\{\operatorname{tr}(M(\lambda)) \geq 2\}$ est de la forme

$$
I=\cup_{k \geq-1} I_{k}
$$

avec $\left.\left.I_{-1}=\right]-\infty, \lambda_{-1}\right], I_{0}=\left[\lambda_{0}, 0\right], I_{k}=\left[\lambda_{k}, \lambda_{k}^{\prime}\right]$ pour $k \geq 1$, avec

$$
\lambda_{-1}<\lambda_{0}<0<\lambda_{1} \leq \lambda_{1}^{\prime}<\lambda_{2} \leq \lambda_{2}^{\prime}<\cdots \text {. }
$$

(iii) Il existe $k \geq 1$ tel que $\lambda_{k} \neq \lambda_{k}^{\prime}$.

(iv) On a $\varlimsup_{\lambda \rightarrow \infty} \operatorname{tr}(M(\lambda))=2$.

Démonstration. - Cette proposition est démontrée dans [5, prop. 4.1] pour les exposants $p \geq 7$; le même argument traite le cas $p=5$. Pour $p=3$, il reste à vérifier que le potentiel $q(t)=-3 a^{2} G^{2}(a t)$, où $a=\Gamma / \pi$ ne vérifie pas l'équation (29) de [4]

$$
q^{\prime \prime}=3 q^{2}+a q+b
$$

avec $a, b$ constantes, ce qui est immédiat.

D'après la proposition 3.1, il existe $\mu_{0}>0$ et $\zeta_{0}>0$ tels que la fonction $\operatorname{tr}\left(M\left(\zeta^{2}\right)\right)$ atteigne son maximum $2 \cosh \left(\Gamma \mu_{0}\right)$ en $\zeta_{0}$. On a en particulier

$$
\mathrm{e}^{\Gamma \mu_{0}}=\sup _{\lambda \geq 0}\{\text { valeurs propres réelles de } M(\lambda)\}>1 .
$$

On peut alors choisir une matrice hermitienne définie positive $\zeta \mapsto Q(\zeta)$, dépendant régulièrement de $\zeta$, telle que, en notant $\mathbb{C}_{\zeta}^{2}$ le plan $\mathbb{C}^{2}$ muni de la norme $\|z\|_{\zeta}^{2}={ }^{t} \bar{z} Q(\zeta) z$, on ait

$$
\begin{aligned}
& \sup _{\zeta \in \mathbb{R}}\left\|M\left(\zeta^{2}\right) ; \mathbb{C}_{\zeta}^{2}\right\|=\mathrm{e}^{\Gamma \mu_{0}}, \\
& \lim _{|\zeta| \rightarrow \infty}\left\|M\left(\zeta^{2}\right) ; \mathbb{C}_{\zeta}^{2}\right\|=1, \\
& Q(\zeta)=\left(\begin{array}{cc}
\zeta^{2} & 0 \\
0 & 1
\end{array}\right) \quad \text { pour } \zeta \text { assez grand. }
\end{aligned}
$$

Soit $\mathbb{T}$ le tore $\mathbb{R} /\left(2 \pi / \zeta_{0} \mathbb{Z}\right)$ et $\mathcal{H}$ l'espace $H^{1}(\mathbb{T}) \oplus L^{2}(\mathbb{T})$ muni de la norme

$$
\left\|f=\left(f_{0}, f_{1}\right)\right\|_{\mathcal{H}}^{2}=\sum_{k}{ }^{t} \bar{f}_{k} Q\left(k \zeta_{0}\right) \widehat{f}_{k}
$$

où

$$
\widehat{g}_{k}=\frac{\zeta_{0}}{2 \pi} \int_{0}^{2 \pi / \zeta_{0}} \mathrm{e}^{-i k z \zeta_{0}} g(z) \mathrm{d} z
$$

TOME $133-2005-\mathrm{N}^{\mathrm{O}} 1$ 
est le $k$-ième coefficient de Fourier de la fonction $2 \pi / \zeta_{0}$ périodique $g$. D'après (3.10), la norme $\left\|f=\left(f_{0}, f_{1}\right)\right\|_{\mathcal{H}}$ est équivalente à la norme usuelle sur $H^{1} \oplus L^{2}$ :

$$
\exists C, \quad C\|f\|_{\mathcal{H}}^{2} \leq \sum_{k}\left(\left(1+k^{2}\right)\left|f_{0, k}\right|^{2}+\left|f_{1, k}\right|^{2}\right) \leq \frac{1}{C}\|f\|_{\mathcal{H}}^{2}
$$

et on a de plus d'après (3.8), l'identité

$$
\|Z(\Gamma) ; \mathcal{H}\|=\mathrm{e}^{\Gamma \mu_{0}} .
$$

Il en résulte pour tout $s>0$

$$
\|Z(s) ; \mathcal{H}\| \leq \mathrm{C}^{\mathrm{te}} \mathrm{e}^{s \mu_{0}} .
$$

Soient $e=\left(e_{0}, e_{1}\right) \in \mathbb{R}^{2} \backslash 0$ vérifiant

$$
M\left(\zeta_{0}^{2}\right) e=\mathrm{e}^{\Gamma \mu_{0}} e
$$

et $\Theta(s)$ la solution de l'équation différentielle ordinaire

$$
\left(\partial_{s}^{2}+p G^{p-1}(s)+\zeta_{0}^{2}\right) \Theta(s)=0, \quad \Theta(0)=e_{0}, \quad \Theta^{\prime}(0)=e_{1} .
$$

La fonction $\Theta(s)$ vérifie

$$
\Theta(s+k \Gamma)=\mathrm{e}^{k \Gamma \mu_{0}} \Theta(s) .
$$

Proposition 3.2. - Il existe $c_{0}>0, c_{1}>0$ tels que pour tout $\left.\left.c \in\right] 0, c_{0}\right]$ et tout $\varepsilon \in] 0, c[$, l'unique solution $v(s, z)$ de l'équation

$$
\left\{\begin{array}{l}
\partial_{s}^{2} v-\partial_{z}^{2} v+v^{p}=0 \\
v(0, z)=1+\varepsilon \cos \left(z \zeta_{0}\right) e_{0}, \quad \partial_{s} v(0, z)=\varepsilon \cos \left(z \zeta_{0}\right) e_{1} .
\end{array}\right.
$$

vérifie

$$
\left\{\begin{array}{l}
v(s, z)=G(s)+\varepsilon\left(\cos \left(z \zeta_{0}\right) \Theta(s)+w(s, z)\right), \\
\left\|w(s, .), H^{1}(\mathbb{T})\right\| \leq c_{1} c \mathrm{e}^{s \mu_{0}}
\end{array}\right.
$$

pour tout $s \geq 0$ tel que $\varepsilon \mathrm{e}^{\mathrm{s} \mu_{0}} \leq$ c. En particulier, on a $w(s, z)=\sum_{k} w_{k}(s) \mathrm{e}^{i k z \zeta_{0}}$,

$$
\left|w_{ \pm 1}(s)\right| \leq c_{1} c \mathrm{e}^{s \mu_{0}} \text {. }
$$

Démonstration. - Remarquons d'abord que l'équation d'onde 1D, non linéaire, (3.16) possède une unique solution globale $\left(v, \partial_{s} v\right)$ continue en $s$ à valeurs dans $\mathcal{H}$. Soient $\mid$. | la norme $\operatorname{sur} C^{0}\left(\left[0, s_{0}\right] ; \mathcal{H}\right)=\mathcal{A}$,

$$
\begin{gathered}
|f|=\sup _{0 \leq s \leq s_{0}} \mathrm{e}^{-\mu_{0} s}\|f(s)\|_{\mathcal{H}} \\
w_{0}(s, z)=\cos \left(z \zeta_{0}\right) \cdot \Theta(s) .
\end{gathered}
$$

D'après (3.14), on a

$$
\left|\left(w_{0}, w_{0}^{\prime}\right)\right| \leq \mathrm{C}^{\mathrm{te}}
$$

BULLETIN DE LA SOCIÉTÉ MATHÉMATIQUE DE FRANCE 
Si $N$ désigne l'opérateur non linéaire

$$
N(g)(s, z)=-\sum_{k=2}^{p} C_{p}^{k} G(s)^{p-k} \varepsilon^{k-1}(g(s, z))^{k},
$$

la fonction $w$ définie par (3.17) vérifie l'équation

$$
L(w)=N\left(w_{0}+w\right), \quad w(0, z)=0, \partial_{s} w(0, z)=0,
$$

d'où

$$
\left(\begin{array}{c}
w(s, z) \\
\partial_{s} w(s, z)
\end{array}\right)=\int_{0}^{s} Z(s-u)\left(\begin{array}{c}
0 \\
N\left(w_{0}+w\right)(u, .)
\end{array}\right) \mathrm{d} u \stackrel{\text { déf }}{=} \Phi\left(w_{0}+w\right) .
$$

L'opérateur $\Phi$, vu comme application de $\mathcal{A}$ dans $\mathcal{A}$ vérifie donc, d'après (3.14), (3.22), (3.24), en utilisant l'injection $H^{1}(\mathbb{T}) \subset L^{\infty}(\mathbb{T})$, avec $\underline{h}=\left(h, \partial_{s} h\right)$, $c=\sup _{0 \leq s \leq s_{0}} \varepsilon \mathrm{e}^{s \mu_{0}}$, et pour $|\underline{h}|+\left|\underline{h}^{\prime}\right| \leq M$,

$$
\left\{\begin{array}{l}
|\Phi(\underline{h})| \leq \mathrm{C}^{\mathrm{te}} c\left(1+(c M)^{p-2}\right) M^{2}, \\
\left|\Phi(\underline{h})-\Phi\left(\underline{h}^{\prime}\right)\right| \leq \mathrm{C}^{\text {te }} c\left(1+(c M)^{p-2}\right)\left|\underline{h}-\underline{h}^{\prime}\right| M .
\end{array}\right.
$$

D'après (3.21) et (3.25), le théorème du point fixe entraîne que l'équation (3.24), $\underline{w}=\Phi\left(w_{0}+w\right)$, possède une unique solution $\underline{w}$ dans $\mathcal{A}$, qui vérifie $|\underline{w}| \leq \mathrm{C}^{\text {te }} c$ dès que $\left.\left.c \in\right] 0, c_{0}\right]$ avec $c_{0}$ assez petit, d'où (3.17).

\section{Preuve du théorème 1}

On reprend les notations du $\S 2$, avec $d^{\prime}=1$ et $d^{\prime \prime}=d-1$. Soit $\left.\sigma \in\right] 1, \sigma_{\text {crit }}[$; choisissons $\delta \in] \sigma+2 /(p-1), d / 2]$. Soit $\tau>0$ petit; on pose

$$
\begin{cases}\alpha_{0}=\min \left(\delta-\sigma, \frac{2 \delta}{p+1}\right), & \alpha=\alpha_{0}-\tau, \\ \beta=\frac{1}{2}(p-1) \alpha-1>0, & \left.\left.\gamma=\frac{2 \delta-1}{d-1} \in\right] 0,1\right] .\end{cases}
$$

Soit $\varphi \in C_{0}^{\infty}\left(R^{d}\right)$, vérifiant

$$
\varphi\left(y_{1}, y^{\prime \prime}\right)= \begin{cases}1 & \text { pour }\left|y_{1}\right| \leq 1 \text { et }\left|y^{\prime \prime}\right| \leq 1 \\ 0 & \text { pour }\left|y_{1}\right| \geq 2 \text { ou }\left|y^{\prime \prime}\right| \geq 2 .\end{cases}
$$

Pour $h \in] 0,1]$, choisissons une fonction $s(h)$ vérifiant

$$
\frac{\operatorname{Ln}(h)}{s(h)} \longrightarrow 0, \quad \forall \nu, h^{\nu} s(h) \longrightarrow 0 \quad(h \rightarrow 0) .
$$

Soient $c>0$ fixé, vérifiant $c \ll \max (\Theta(s), s \in[0, \Gamma])$ (voir la proposition $3.2)$, et $\varepsilon(h) \in O\left(h^{\infty}\right)$ défini par

$$
\varepsilon(h) \mathrm{e}^{\mu_{0} s(h)}=c .
$$

TOME $133-2005-\mathrm{N}^{\mathrm{O}} 1$ 
Pour $h \in] 0,1]$, soit $v(s, y, h)$ une solution faible de l'équation

$$
\left\{\begin{array}{l}
\partial_{s}^{2} v-h^{2 \beta}\left(\triangle_{y_{1}}+h^{2(1-\gamma)} \triangle_{y^{\prime \prime}}\right) v+v^{p}=0, \\
v(0, y, h)=\varphi(y)\left(1+\varepsilon(h) \cos \left(y_{1} \zeta_{0} / h^{\beta}\right) e_{0}=v_{0, h}(y),\right. \\
\partial_{s} v(0, y, h)=\varphi(y) \varepsilon(h) \cos \left(y_{1} \zeta_{0} / h^{\beta}\right) e_{1}=v_{1, h}(y) .
\end{array}\right.
$$

La vitesse de propagation de l'équation (4.5) étant majorée par $h^{\beta}$, la fonction $v(s, y, h)$ coïncide dans le domaine $|y|<\frac{1}{2}$ et pour $|s|<s(h)$, avec l'unique solution de l'équation (4.5) dans laquelle on a remplacé $\varphi$ par 1 dans les données de Cauchy de (4.5) (on a $\beta>0$, donc $s(h) h^{\beta} \ll 1$ d'après (4.3)).

D'après la proposition 3.2, il en résulte dans le domaine $|y|<\frac{1}{2}$ et $|s|<s(h)$

$$
\left\{\begin{array}{l}
v(s, y, h)=b\left(s, y_{1}, h\right), \\
b\left(s, y_{1}, h\right)=G(s)+\varepsilon(h)\left(\cos \left(y_{1} \zeta_{0} / h^{\beta}\right) \Theta(s)+w\left(s, y_{1} / h^{\beta}\right)\right), \\
\left\|w(s, .), H^{1}(\mathbb{T})\right\| \leq c_{1} c \mathrm{e}^{s \mu_{0}} .
\end{array}\right.
$$

Posons, pour $h>0$ petit,

$$
u_{h, \tau}\left(t, x^{\prime}, x^{\prime \prime}\right)=h^{-\alpha} v\left(\frac{t}{h^{1+\beta}}, \frac{x^{\prime}}{h}, \frac{x^{\prime \prime}}{h^{\gamma}}\right) .
$$

La fonction $u_{h, \tau}$ vérifie l'équation (1.1), et puisque $\varphi \in C_{0}^{\infty}$ et $\varepsilon(h) \in O\left(h^{\infty}\right)$, la famille $\left(u_{h, \tau}(0, x), \partial_{t} u_{h, \tau}(0, x)\right)$ est $O\left(h^{\tau}\right)$ dans $\left(H_{0}^{\sigma} \cap L^{p+1} \times C_{0}^{\infty}\right)$. D'après $(2.5)$, pour tout $\sigma^{\prime}$, on a

$$
\left\|u_{h, \tau}(t, .) ; H^{\sigma^{\prime}}\right\| \geq h^{\delta-\alpha-\sigma^{\prime}}\left(\int\left(h^{2}+\left|\eta_{1}\right|^{2}\right)^{\sigma^{\prime}}\left|\widehat{v}\left(\frac{t}{h^{1+\beta}}, \eta\right)\right|^{2} \mathrm{~d} \eta\right)^{1 / 2} .
$$

D'après la proposition (3.2) et le choix des fonctions $\varepsilon(h), s(h)$, il en résulte la Proposition 4.1. - Il existe $h_{0}>0, c_{2}>0$ et, pour $\left.\left.h \in\right] 0, h_{0}\right]$, un temps $t(h)$ vérifiant

$$
\forall M, \forall \nu, \quad M h^{1+\beta} \log (h)<t(h)<h^{1+\beta-\nu} \quad(h \rightarrow 0)
$$

et telle qu'on ait, pour tout $\sigma^{\prime} \geq 0$, et pour toute solution faible $v$ de (4.5)

$$
\left\|u_{h, \tau}(t(h), .) ; H^{\sigma^{\prime}}\right\| \geq c_{2} h^{\delta-\alpha-\sigma^{\prime}(1+\beta)}
$$

où $u_{h, \tau}$ est défini par (4.7).

Démonstration. - La proposition résulte de (3.15), (4.4), (4.6), (4.8) et de

$$
\left.\int\left|\eta_{1}\right|^{2 \sigma^{\prime}}\left|\widehat{v}\left(\frac{t}{h^{1+\beta}}, \eta\right)\right|^{2} \mathrm{~d} \eta\right)+O\left(h^{\infty}\right) \geq \mathrm{C}^{\mathrm{te}} \int_{\left|\eta_{1}-\zeta_{0} / h^{\beta}\right| \leq 1}\left|\eta_{1}\right|^{2 \sigma^{\prime}}\left|\widehat{\psi} b\left(\frac{t}{h^{1+\beta}}, \eta\right)\right|^{2} d \eta
$$

où $\psi(y) \in C_{0}^{\infty}$ localise dans $|y|<\frac{1}{2}$. 
On achève alors la preuve du théorème 1 en construisant les données de Cauchy $u_{0}, u_{1}$ comme superposition de données de Cauchy de translatéeshomogénéisées des $v_{., h}$ comme suit.

On choisit $\delta=\frac{1}{2} d$ pour $\sigma \geq \sigma_{\text {sob }}$ et $\delta=\sigma(p+1) /(p-1)$ pour $\sigma \leq \sigma_{\text {sob }}$. Dans les deux cas, on a $\alpha_{0}=\delta-\sigma$ et la condition $\sigma^{\prime}(1+\beta)>\sigma$ équivaut à $\sigma^{\prime}>I(\sigma)$.

Posons $h_{n}=2^{-n}, \tau_{n}=1 / \sqrt{n}, \alpha_{n}=\alpha_{0}-\tau_{n}, \beta_{n}=\frac{1}{2}(p-1) \alpha_{n}-1$ et $x_{n}=(1 / n, 0, \ldots, 0) \in \mathbb{R}^{d}$. On définit les donnés de Cauchy $u_{0}, u_{1}$ par (avec $n_{0}$ fixé assez grand)

$$
\left\{\begin{array}{l}
u_{0}=\sum_{n>n_{0}} u_{0, n}, \quad u_{0, n}=h_{n}^{-\alpha_{n}} v_{0, h_{n}}\left(\frac{x_{1}-n^{-1}}{h_{n}}, \frac{x^{\prime \prime}}{h_{n}^{\gamma}}\right) \\
u_{1}=\sum_{n>n_{0}} u_{1, n}, \quad u_{1, n}=h_{n}^{-\left(\alpha_{n}+1+\beta_{n}\right)} v_{1, h_{n}}\left(\frac{x_{1}-n^{-1}}{h_{n}}, \frac{x^{\prime \prime}}{h_{n}^{\gamma}}\right) .
\end{array}\right.
$$

La série $\sum_{n} h_{n}^{\tau_{n}}$ étant convergente, on a $\left(u_{0}, u_{1}\right) \in H_{0}^{\sigma} \cap L^{p+1} \times C_{0}^{\infty}$.

Soit $u(t, x)$ une solution faible de (1.1), (1.2), de données de Cauchy $\left(u_{0}, u_{1}\right)$ et $t_{k}=t\left(h_{k}\right)$. Comme pour $k$ grand on a

$$
\operatorname{dist}\left(x_{k}, x_{k \pm 1}\right)>\frac{1}{2 k^{2}}>2\left(3 h_{k}^{\gamma}+t_{k}\right)
$$

par vitesse finie de propagation, la fonction $u\left(t_{k}, x\right)$ coïncide dans la boule de centre $x_{k}$ et de rayon $1 / 10 k^{2}$ avec une fonction du type $u_{h_{k}, \tau_{k}}\left(t_{k}, x\right)$, et il existe une fonction $\psi(x) \in C_{0}^{\infty}$ telle que

$$
\psi\left(\frac{x-x_{k}}{k^{2}}\right) u\left(t_{k}, x\right)=u_{h_{k}, \tau_{k}}\left(t_{k}, x\right)
$$

Il en résulte d'après la proposition 4.1, qu'il existe un polynôme pol $(k)$ tel qu'on ait

$$
\left\|u\left(t_{k}, .\right) ; H^{\sigma^{\prime}}\right\| \operatorname{pol}(k) \geq 2^{-k\left(\sigma-\sigma^{\prime}(1+\beta)\right)} 2^{\sqrt{k}} .
$$

D'où le théorème 1 .

\section{Preuve du théorème 2}

La preuve du théorème 2 étant analogue à celle du théorème 1 , nous ne préciserons que les variantes principales.

On choisit $G_{1}$ solution de

$$
G_{1}^{\prime \prime}+G_{1}^{p}=0, \quad G_{1}(0)=0, G_{1}^{\prime}(0)=1 .
$$

On a $G_{1}(s)=a^{2 /(p-1)} G\left(a\left(s+s_{0}\right)\right), a^{2}=\left(\frac{1}{2}(p+1)\right)^{\frac{p-1}{p+1}}$ et on note $\Theta_{1}, \mu_{1}, \zeta_{1}$ les fonctions et les réels associés à $G_{1}$ comme dans le $\S 3$. 
On choisit $\delta=\frac{1}{2} d, \gamma=1$ et on note cette fois $v$ la solution de

$$
\left\{\begin{array}{l}
\partial_{s}^{2} v-h^{2 \beta} \triangle_{y} v+v^{p}=0 \\
v(0, y, h)=\varphi(y)\left(\varepsilon(h) \cos \left(y_{1} \zeta_{1} / h^{\beta}\right) e_{0}\right. \\
\partial_{s} v(0, y, h)=\varphi(y)\left(1+\varepsilon(h) \cos \left(y_{1} \zeta_{1} / h^{\beta}\right) e_{1}\right) .
\end{array}\right.
$$

On a comme précédemment dans le domaine $|y|<\frac{1}{2}$ et $|s|<s(h)$

$$
\left\{\begin{array}{l}
v(s, y, h)=b\left(s, y_{1}, h\right) \\
b\left(s, y_{1}, h\right)=G_{1}(s)+\varepsilon(h)\left(\cos \left(y_{1} \zeta_{1} / h^{\beta}\right) \Theta_{1}(s)+w\left(s, y_{1} / h^{\beta}\right)\right), \\
\left\|\partial_{s} w(s, .), L^{2}(\mathbb{T})\right\| \leq c_{1} c \mathrm{e}^{s \mu_{1}}
\end{array}\right.
$$

Posons à nouveau

$$
u_{h}(t, x)=h^{-\alpha} v\left(\frac{t}{h^{1+\beta}}, \frac{x}{h}\right) .
$$

Alors d'après (2.5), la famille $\left(u_{h}(0, x), \partial_{t} u_{h}(0, x)\right)$ est $O(1)$ dans $C_{0}^{\infty} \times H_{0}^{\sigma-1}$ sous la condition $\delta-\alpha-\sigma-\beta=0$ et on a

$$
\begin{aligned}
& \left\|\partial_{t} u_{h}(t, .) ; H^{\sigma^{\prime}-1}\right\| \\
& \quad \geq h^{\delta-\alpha-\sigma^{\prime}-\beta}\left(\int\left(h^{2}+\left|\eta_{1}\right|^{2}\right)^{\sigma^{\prime}-1}\left|\widehat{\partial_{s} v}\left(\frac{t}{h^{1+\beta}}, \eta\right)\right|^{2} \mathrm{~d} \eta\right)^{1 / 2}
\end{aligned}
$$

quantité non bornée quand $h \rightarrow 0$ dès que

$$
\delta-\alpha-\sigma^{\prime}-\beta \sigma^{\prime}<0
$$

donc pour tout $\sigma^{\prime}>J(\sigma)$ d'après la définition de $J$ et (2.9). D'où le théorème 2 .

\section{Appendice : solutions faibles}

Nous rappelons dans cet appendice la méthode de construction de solution faible au problème de Cauchy (1.1), (1.2).

Soit $F_{\epsilon}(u)$ une famille de symboles en $u$ de degré $2, C^{\infty}$, positifs, convexes, pairs, qui converge vers $u^{p+1} /(p+1)$ quand $\epsilon$ tend vers 0 , et dont la dérivée (au plus linéaire à l'infini) $f_{\epsilon}(u)=F_{\epsilon}^{\prime}(u)$ converge en croissant vers $u^{p}$ et vérifie pour tout $u, h$

$$
\left|f_{\epsilon}(u+h)-f_{\epsilon}(u)\right| \leq C\left(\left|f_{\epsilon}(h)\right|+|u|^{p-1} \cdot\left|f_{\epsilon}(h)\right|^{1 / p}\right) .
$$

On peut choisir par exemple pour $F_{\epsilon}(u)$ la primitive nulle en 0 de la fonction

$$
f_{\epsilon}(u)=\frac{u^{p}}{1+(\epsilon u)^{p-1}} .
$$

Soit $u_{\epsilon}(t, x)$ l'unique solution globale du problème de Cauchy

$$
\begin{gathered}
\left(\partial_{t}^{2}-\triangle_{x}\right) u_{\epsilon}+f_{\epsilon}\left(u_{\epsilon}\right)=0, \\
\left.u_{\epsilon}(0, x)=u_{0}(x), \quad \partial_{t} u_{\epsilon}(0, x)\right)=u_{1}(x),
\end{gathered}
$$

BULlETIN DE LA SOCIÉTÉ MATHÉMATIQUE DE FRANCE 
où les données de Cauchy $u_{0}, u_{1}$ sont fixées dans l'espace d'énergie $\dot{H}^{1} \cap L^{p+1} \times L^{2}$. La fonction $u_{\epsilon}(t, x)$ vérifie l'estimation d'énergie

$$
\int \frac{1}{2}\left(\left|\partial_{t} u\right|_{\epsilon}^{2}+\left|\nabla_{x} u_{\epsilon}\right|^{2}\right)+F_{\epsilon}\left(u_{\epsilon}\right) \mathrm{d} x=\mathrm{C}^{\mathrm{te}} .
$$

Il résulte de (6.2), (6.4) et du lemme de Fatou qu'on peut extraire de la famille $u_{\epsilon}(t, x)$ une suite convergeant dans l'espace $C_{t}^{1}\left(\mathcal{D}_{x}^{\prime}\right)$ et pour presque tout $(t, x)$, vers une fonction $u(t, x) \in L^{\infty}\left(t, \dot{H}^{1} \cap L^{p+1}\right)$.

Pour vérifier que $u$ satisfait le problème de Cauchy (1.1), (1.2), il suffit alors de montrer que pour tout $\phi(t, x) \in C_{0}^{\infty}$, on a

$$
\lim \int f_{\epsilon}\left(u_{\epsilon}\right) \phi \mathrm{d} t \mathrm{~d} x=\int u^{p} \phi \mathrm{d} t \mathrm{~d} x .
$$

Or on a par convergence dominée

$$
\int\left|\left(u^{p}(t, x)-f_{\epsilon}(u(t, x))\right) \phi(t, x)\right| \mathrm{d} t \mathrm{~d} x \longrightarrow 0 .
$$

Soit $h_{\epsilon}=u_{\epsilon}-u$. D'après (6.4), on peut supposer que la famille $h_{\epsilon}$ converge fortement vers 0 dans $L_{\text {loc }}^{2}$. D'après l'inégalité de Hölder, en remarquant que l'on a $\left|f_{\epsilon}(h)\right| \leq \mathrm{C}^{\text {te }}|h|^{\alpha} F_{\epsilon}(h)^{\beta}$ avec $\alpha=2 /(p-1)$ et $\beta=(p-2) /(p-1)$, on obtient

$$
\int\left|f_{\epsilon}\left(h_{\epsilon}\right) \phi\right| \mathrm{d} t \mathrm{~d} x \longrightarrow 0
$$

D'après (5.1), on a de plus

$$
\int\left|\left(f_{\epsilon}\left(u_{\epsilon}\right)-f_{\epsilon}(u)\right) \phi\right| \mathrm{d} t \mathrm{~d} x \leq C \int\left(\left|f_{\epsilon}\left(h_{\epsilon}\right)\right|+u^{p-1}\left|f_{\epsilon}\left(h_{\epsilon}\right)\right|^{1 / p} \mid\right)|\phi| \mathrm{d} t \mathrm{~d} x
$$

de sorte que (6.5) résulte de (6.6), (6.7), (6.8) et de l'inégalité de Hölder.

\section{BIBLIOGRAPHIE}

[1] Brenner (P.) \& VON WAHL (W.) - Global classical solutions of non-linear wave equations, Math. Z., t. 176 (1981), pp. 87-121.

[2] Ginibre (J.) \& Velo (G.) - The global Cauchy problem for the non linear Klein-Gordon equation, Math.Z., t. 189 (1985), pp. 487-505.

[3] Hochstadt (H.) - On the Determination of a Hill's Equation from its Spectrum, Arch. Rat. Mech. Anal., t. 19 (1965), pp. 353-362.

[4] Jörgens (K.) - Das Anfangswertproblem in Grossen fur eine Klasse nichtlinearer Wellingleichungen, Math. Z., t. 77 (1961), pp. 295-308.

[5] LeBeau (G.) - Non linear optic and supercritical wave equation, Bull. Soc. Math. Liège, t. 70 (2001), pp. 267-306.

[6] Lions (J.-L.) - Quelques méthodes de résolution des problèmes aux limites non-linéaires, Dunod Gauthier-Villars, Paris, 1969. 
[7] Segal (I.E) - The Global Cauchy Problem for a Relativistic Scalar Field with Power Interaction, Bull. Soc. Math. France, t. 91 (1963), pp. 129-135.

[8] Shatah (J.) \& Struwe (M.) - Well-Posedness in the Energy Space for Semilinear Wave Equation with Critical Growth, I.M.R.N., t. 7 (1994), pp. 303-309.

[9] Strauss (W.) - Nonlinear invariant wave equations, in Invariant wave equations (Proc. "Ettore Majorana" Internat. School of Math. Phys., Erice, 1977), Lecture Notes in Phys., vol. 73, Springer, 1978, pp. 197-249. 